\title{
Bicomplex holomorphic functional calculus
}

\author{
Fabrizio Colombo ${ }^{* * 1}$, Irene Sabadini ${ }^{* 1}$, and Daniele C. Struppa ${ }^{* * 2}$ \\ ${ }^{1}$ Politecnico di Milano, Dipartimento di Matematica, Via Bonardi 9, 20133, Milano, Italy \\ ${ }^{2}$ Schmid College of Science and Technology, Chapman University, Orange, CA 92866, USA
}

Received 26 December 2012, revised 25 September 2013, accepted 29 September 2013

Published online 11 December 2013

Key words Bicomplex holomorphic analysis, bounded bicomplex linear operators, bicomplex holomorphic functional calculus, reduced spectrum

MSC (2010) 30G35, 47A10, 47A60

In this paper we introduce and study a functional calculus for bicomplex linear bounded operators. The study is based on the decomposition of bicomplex numbers and of linear operators using the two nonreal idempotents. We show that, due to the presence of zero divisors in the bicomplex numbers, the spectrum of a bounded operator is unbounded. We therefore introduce a different spectrum (called reduced spectrum) which is bounded and turns out to be the right tool to construct the bicomplex holomorphic functional calculus. Finally we provide some properties of the calculus.

\section{Introduction}

A functional calculus is a theory allowing one to construct from a given function $f$ and a given operator $T$ a linear operator $f(T)$ that depends on the function $f$. This is a branch of the field of functional analysis, connected with spectral theory of operators.

When $f$ is a function of a real variable $x$, for example, and $T$ is an operator, in general the expression $f(T)$ does not make sense. If it does, then we are no longer using $f$ on its original function domain.

If a given function $f$ is of certain special type, there are natural ways of defining $f(T)$. For instance, if $f$ is a complex polynomial $f(z)=\sum_{j=0}^{n} a_{j} z^{j}$, one can simply substitute $T$ for $z$ and write $f(T)=\sum_{j=0}^{n} a_{j} T^{j}$. This is the polynomial functional calculus. If $f$ is an entire function, with series expansion $f(z)=\sum_{j=0}^{\infty} a_{j} z^{j}$, we can define $f(T)=\sum_{j=0}^{\infty} a_{j} T^{j}$ whenever $T$ is a bounded operator. Since the Mac Laurin series converges everywhere, the above series will converge as well, in a suitable operator norm. The requirement that the Mac Laurin series of $f$ converges everywhere can be relaxed, we will not enter into the details here. It is expected that a necessary condition for $f(T)$ to make sense is $f$ be defined on the spectrum of T. Other indications also reinforce the idea that $f(T)$ can be defined only if $f$ is defined on the spectrum of $T$. The most familiar functional calculus is the holomorphic functional calculus and it is based on the Cauchy integral formula from the classical theory of a complex variable. In the Cauchy's integral formula we replace the Cauchy kernel by the resolvent operators $(\lambda I-T)^{-1}$ and we integrate on a contour $\Gamma$, which is the boundary of a suitably smooth domain $U$ that contains the spectrum of $T$, which, in the case of bounded operators, in a compact and nonempty set.

Assuming that the Banach space-valued integral is appropriately defined, this proposed functional calculus implies the following necessary condition: the functional calculus should be well-defined in the sense that $f(T)$ is independent of $\Gamma$.

In the definition of the functional calculus, $f$ is assumed to be holomorphic in an open neighborhood of $U$ and it turns out that the resolvent mapping is holomorphic on the resolvent set. Therefore the integral makes sense.

The holomorphic functional calculus has been extended in several directions using hypercomplex analysis. Two main areas of investigation are the so-called $S$-functional calculus, based on slice hyperholomorphic functions,

\footnotetext{
* Corresponding author: e-mail: irene.sabadini@ polimi.it, Phone: +39 0223994581, Fax: +39 0223994513

** e-mail: fabrizio.colombo@ @olimi.it, Phone: +39 0223994613, Fax: +39 0223994621

*** e-mail: struppa@ chapman.edu, Phone: + 17149976826
} 
which is the a natural extension of the holomorphic functional calculus to quaternionic operators and to $n$-tuples of noncommuting operators (see the book [4]), and the monogenic functional calculus based on the classical theory of monogenic functions (see the book [8]). The theory of several complex variables gives rise to a functional calculus that is based on the joint spectrum of operators.

The analysis of bicomplex holomorphic functions started in the thirties with the works of Scorza Dragoni and Spampinato, see [18], [19]. In recent times there has been a resurgence of interest for this topic, see e.g. the monograph [13], as well as the articles [5], [6], [7], [14], and its applications, see e.g. [2], [3], [9]. Motivated by these papers, the aim of this article is to investigate the functional calculus in the bicomplex holomorphic setting. We will show that, unlike what happens in the complex case, bounded operators have an unbounded spectrum, see Corollary 3.11; this fact makes it necessary for us to identify a different notion of spectrum, what we will call the reduced spectrum, and we will show how the use of this different notion allows us to define a functional calculus for which it is possible to obtain the main usual properties of a functional calculus.

The plan of the paper is as follows: In Section 2 we recall some basic facts of bicomplex analysis and the Cauchy formula for bicomplex holomorphic functions. In Section 3 we study the spectrum of bounded linear operators and we prove the crucial Theorem 3.10 in which we study the structure of the spectrum of a bounded operator. We then use this result to suitably modify the definition of spectrum in order to get a bounded spectrum, called the reduced spectrum, for bounded operators. We conclude the section and the paper with some properties for this functional calculus.

\section{Preliminary results in bicomplex analysis}

In this section we summarize the main properties of bicomplex numbers and of holomorphic functions of bicomplex numbers, and we refer the reader to [5], [10], [11], [12], [13], [14] for further details.

Let $\mathbf{i}_{1}$ and $\mathbf{i}_{2}$ be two commuting imaginary units (i.e. $\mathbf{i}_{1}^{2}=\mathbf{i}_{2}^{2}=-1$, and $\mathbf{i}_{1} \mathbf{i}_{2}=\mathbf{i}_{2} \mathbf{i}_{1}$ ). By $\mathbb{C}_{\mathbf{i}_{1}}$ and $\mathbb{C}_{\mathbf{i}_{2}}$ we denote, respectively, the field of complex numbers of the form $x+\mathbf{i}_{1} y$ and of the form $x+\mathbf{i}_{2} y, x, y \in \mathbb{R}$. The set of bicomplex numbers $\mathbb{B} \mathbb{C}$ is then defined as

$$
\begin{aligned}
\mathbb{B C} & =\left\{Z=x_{0}+\mathbf{i}_{1} x_{1}+\mathbf{i}_{2} x_{2}+\mathbf{i}_{1} \mathbf{i}_{2} x_{3}, \text { with } x_{0}, x_{1}, x_{2}, x_{3} \in \mathbb{R}\right\} \\
& =\left\{Z=z+\mathbf{i}_{2} w, \text { with } z, w \in \mathbb{C}_{\mathbf{i}_{1}}\right\} .
\end{aligned}
$$

An element $Z \in \mathbb{B C}$ will be called a bicomplex number. The set $\mathbb{B C}$ turns out to be a ring with respect to the sum defined by $Z_{1}+Z_{2}=\left(z_{1}+\mathbf{i}_{2} w_{1}\right)+\left(z_{2}+\mathbf{i}_{2} w_{2}\right)=\left(z_{1}+z_{2}\right)+\mathbf{i}_{2}\left(w_{1}+w_{2}\right)$ and the product $Z_{1} Z_{2}=$ $\left(z_{1}+\mathbf{i}_{2} w_{1}\right)\left(z_{2}+\mathbf{i}_{2} w_{2}\right)=\left(z_{1} z_{2}-w_{1} w_{2}\right)+\mathbf{i}_{2}\left(w_{1} z_{2}+z_{1} w_{2}\right)$ and thus it turns out to be a module over itself. The rings $\mathbb{C}_{\mathbf{i}_{1}}, \mathbb{C}_{\mathbf{i}_{2}}$ are subrings of $\mathbb{B C}$, which are in fact fields, and thus $\mathbb{B C}$ can be seen as vector space over $\mathbb{C}_{\mathbf{i}_{1}}$ and over $\mathbb{C}_{\mathbf{i}_{2}}$.

Let $U \subseteq \mathbb{B C}$ be an open set. A function $F: U \subseteq \mathbb{B C} \rightarrow \mathbb{B C}$ can be written as

$$
F=f_{0}+\mathbf{i}_{1} f_{1}+\mathbf{i}_{2} f_{2}+\mathbf{i}_{1} \mathbf{i}_{2} f_{3},
$$

where $f_{i}: U \subseteq \mathbb{B C} \rightarrow \mathbb{R}$, or as $F=u+\mathbf{i}_{2} v$, where $u=f_{0}+\mathbf{i}_{1} f_{1}$ and $v=f_{2}+\mathbf{i}_{1} f_{3}$.

The algebra $\mathbb{B C}$ is not a division algebra, since one can see that if

$$
\mathbf{e}_{1}=\frac{1+\mathbf{i}_{1} \mathbf{i}_{2}}{2} \quad \text { and } \quad \mathbf{e}_{2}=\frac{1-\mathbf{i}_{1} \mathbf{i}_{2}}{2},
$$

then $\mathbf{e}_{1} \cdot \mathbf{e}_{2}=0$. The following two results are immediate and well known, see e.g. [13].

Proposition 2.1 The bicomplex numbers $\mathbf{e}_{1}, \mathbf{e}_{2}$ are linearly independent, mutually annihilating, idempotent, and satisfy $\mathbf{e}_{1}+\mathbf{e}_{2}=1$.

Proposition 2.2 Every $Z=z+\mathbf{i}_{2} w \in \mathbb{B C}$, where $z, w \in \mathbb{C}_{\mathbf{i}_{1}}$, can be written in a unique way as:

$$
Z=\left(z-\mathbf{i}_{1} w\right) \mathbf{e}_{1}+\left(z+\mathbf{i}_{1} w\right) \mathbf{e}_{2}
$$

and therefore the multiplications by $\mathbf{e}_{1}$ and $\mathbf{e}_{2}$ define two projection operators $P_{1}$ and $P_{2}$ from $\mathbb{B} \mathbb{C}$ to $\mathbb{C}_{\mathbf{i}_{1}}$, both seen as $\mathbb{C}_{\mathbf{i}_{1}}$-vector spaces. 
Formula (2.1) is called the idempotent representation of a bicomplex number. Note that its uniqueness is a consequence of the fact we have required the coefficients of $\mathbf{e}_{1}$ and $\mathbf{e}_{2}$ to be taken in $\mathbb{C}_{\mathbf{i}_{1}}$. A similar representation could have been obtained by taking such coefficients in $\mathbb{C}_{\mathbf{i}_{2}}$.

Remark 2.3 The sets:

$$
A_{1}:=\left\{z-\mathbf{i}_{1} w \mid z, w \in \mathbb{C}_{\mathbf{i}_{1}}\right\}, \quad A_{2}:=\left\{z+\mathbf{i}_{1} w \mid z, w \in \mathbb{C}_{\mathbf{i}_{1}}\right\},
$$

are such that

$$
\mathbb{B C}=\mathbf{e}_{1} A_{1}+\mathbf{e}_{2} A_{2} .
$$

Moreover, for any open set $U \subseteq \mathbb{B} \mathbb{C}$ this decomposition induces two open sets $U_{1} \subseteq A_{1}$ and $U_{2} \subseteq A_{2}$ such that

$$
U=\mathbf{e}_{1} U_{1}+\mathbf{e}_{2} U_{2} .
$$

Remark 2.4 Note that $A_{1}$ and $A_{2}$ are copies of the same complex plane $\mathbb{C}_{\mathbf{i}_{1}}$ but since the variable in $A_{1}$ differs from the variable in $A_{2}$, it is convenient to keep the different notation.

Since bicomplex numbers are defined by using two different imaginary units, there are three natural notions of conjugation.

Definition 2.5 Let $Z=x_{0}+\mathbf{i}_{1} x_{1}+\mathbf{i}_{2} x_{2}+\mathbf{i}_{1} \mathbf{i}_{2} x_{3}=z+\mathbf{i}_{2} w \in \mathbb{B C}$. We define the following three conjugates in $\mathbb{B C}$ :

$$
\begin{aligned}
Z^{*} & =\bar{z}-\mathbf{i}_{2} \bar{w}, \\
\widetilde{Z} & =\bar{z}+\mathbf{i}_{2} \bar{w}, \\
Z^{\dagger} & =z-\mathbf{i}_{2} w .
\end{aligned}
$$

Any bicomplex number $Z=z+\mathbf{i}_{2} w$ such that $Z \cdot Z^{\dagger}=z^{2}+w^{2} \neq 0$ is invertible since the non-zero complex number $z^{2}+w^{2} \in \mathbb{C}_{\mathbf{i}_{1}}$ has a natural complex inverse. In particular, the bicomplex number

$$
Z^{-1}=\frac{Z^{\dagger}}{z^{2}+w^{2}}
$$

satisfies $Z \cdot Z^{-1}=Z^{-1} \cdot Z=1$ and therefore it is the inverse of $Z$. Any bicomplex number for which $Z \cdot Z^{\dagger} \neq 0$ will be said to be nonsingular (or invertible). Let us set

$$
\mathcal{J}_{\ell}:=\mathbf{e}_{\ell} \mathbb{B} \mathbb{C}, \quad \ell=1,2 .
$$

Then it is easy to verify that $\mathcal{J}_{\ell}, \ell=1,2$, is an ideal in $\mathbb{B C}$ and also that a product of two nonzero elements $Z$, $W$ is zero if and only if $Z \in \mathcal{J}_{1}$ and $W \in \mathcal{J}_{2}$.

A simple computation shows that, in terms of the idempotent representation, the inverse of a bicomplex number $Z=z+\mathbf{i}_{2} w \notin \mathcal{J}_{1} \cup \mathcal{J}_{2}$ is given by:

$$
Z^{-1}=\mathbf{e}_{1}\left(z-\mathbf{i}_{1} w\right)^{-1}+\mathbf{e}_{2}\left(z+\mathbf{i}_{1} w\right)^{-1} .
$$

Let us define the Euclidean norm on $\mathbb{B C}$ as

$$
\left|x_{0}+\mathbf{i}_{1} x_{1}+\mathbf{i}_{2} x_{2}+\mathbf{i}_{1} \mathbf{i}_{2} x_{3}\right|:=\left(x_{0}^{2}+x_{1}^{2}+x_{2}^{2}+x_{3}^{2}\right)^{1 / 2} ;
$$

it is immediate to see that such a norm does not respect the multiplicative structure of $\mathbb{B} \mathbb{C}$, since, if $Z, W \in \mathbb{B} \mathbb{C}$, we have (see [13], p. 7):

$$
|Z W| \leq \sqrt{2}|Z||W| ;
$$

however, if $Z \in \mathbb{C}_{\mathbf{i}_{1}}$ then $|Z W|=|Z||W|$. The issue of the various notions of norm in $\mathbb{B C}$ has been widely discussed in [1], Section 1.3. 
In order to study the notion of bicomplex holomorphicity we introduce some very natural differential operators:

$$
\begin{aligned}
\frac{\partial}{\partial Z^{*}} & :=\frac{\partial}{\partial x_{0}}+\mathbf{i}_{1} \frac{\partial}{\partial x_{1}}+\mathbf{i}_{2} \frac{\partial}{\partial x_{2}}+\mathbf{i}_{1} \mathbf{i}_{2} \frac{\partial}{\partial x_{3}}=\frac{\partial}{\partial \bar{z}}+\mathbf{i}_{2} \frac{\partial}{\partial \bar{w}}, \\
\frac{\partial}{\partial \tilde{Z}} & :=\frac{\partial}{\partial x_{0}}+\mathbf{i}_{1} \frac{\partial}{\partial x_{1}}-\mathbf{i}_{2} \frac{\partial}{\partial x_{2}}-\mathbf{i}_{1} \mathbf{i}_{2} \frac{\partial}{\partial x_{3}}=\frac{\partial}{\partial \bar{z}}-\mathbf{i}_{2} \frac{\partial}{\partial \bar{w}}, \\
\frac{\partial}{\partial Z^{\dagger}} & :=\frac{\partial}{\partial x_{0}}-\mathbf{i}_{1} \frac{\partial}{\partial x_{1}}+\mathbf{i}_{2} \frac{\partial}{\partial x_{2}}-\mathbf{i}_{1} \mathbf{i}_{2} \frac{\partial}{\partial x_{3}}=\frac{\partial}{\partial z}+\mathbf{i}_{2} \frac{\partial}{\partial w}, \\
\frac{\partial}{\partial Z} & :=\frac{\partial}{\partial x_{0}}-\mathbf{i}_{1} \frac{\partial}{\partial x_{1}}-\mathbf{i}_{2} \frac{\partial}{\partial x_{2}}+\mathbf{i}_{1} \mathbf{i}_{2} \frac{\partial}{\partial x_{3}}=\frac{\partial}{\partial z}-\mathbf{i}_{2} \frac{\partial}{\partial w} .
\end{aligned}
$$

Let $U \subseteq \mathbb{B C}$ be an open set, and let $F: U \subseteq \mathbb{B C} \rightarrow \mathbb{B C}$ be a bicomplex function, written as $F=u+\mathbf{i}_{2} v$, where $u, v: U \subseteq \mathbb{B C} \rightarrow \mathbb{C}_{\mathbf{i}_{1}}$. We define bicomplex holomorphicity in the same way one defines classical holomorphicity in one complex variable:

Definition 2.6 (See [12], [13].) A function is said to be bicomplex holomorphic in an open set $U \subseteq \mathbb{B} \mathbb{C}$ if it admits a bicomplex derivative at each point, i.e. if the limit

$$
F^{\prime}\left(Z_{0}\right):=\lim _{\substack{Z \rightarrow Z_{0} \\ Z-Z_{0} \text { inv }}}\left(Z-Z_{0}\right)^{-1}\left(F(Z)-F\left(Z_{0}\right)\right)
$$

exists and is finite for any $Z_{0}$ in $U$ such that $Z-Z_{0}$ is invertible. The limit will be called the derivative of $F$ and denoted by $F^{\prime}\left(Z_{0}\right)$.

A thorough discussion of derivability and differentiability for bicomplex functions can be found in [12].

We know that holomorphic functions in one complex variable are solutions of the Cauchy-Riemann system. Something similar occurs in this case.

Theorem 2.7 (See [13].) Let $U$ be an open set and $F: U \subseteq \mathbb{B} \mathbb{C} \rightarrow \mathbb{B C}$ such that $F=u+\mathbf{i}_{2} v \in C^{1}(U)$. Then $F$ is bicomplex holomorphic if and only if:

(1) $u$ and $v$ are complex holomorphic in $z$ and $w$,

(2) $\frac{\partial u}{\partial z}=\frac{\partial v}{\partial w}$ and $\frac{\partial v}{\partial z}=-\frac{\partial u}{\partial w}$ on $U$.

Moreover, $F^{\prime}=\frac{1}{2} \frac{\partial F}{\partial Z}=\frac{\partial u}{\partial z}+\mathbf{i}_{2} \frac{\partial v}{\partial z}=\frac{\partial v}{\partial w}-\mathbf{i}_{2} \frac{\partial u}{\partial w}$.

The next result is the foundation for the theory of bicomplex holomorphic functions, and for the understanding of their behavior:

Theorem 2.8 Let $U \subseteq \mathbb{B C}$ be an open set and let $F: U \subseteq \mathbb{B C} \rightarrow \mathbb{B C}$ be such that $F=u+\mathbf{i}_{2} v \in C^{1}(U)$. Then $F$ is bicomplex holomorphic if and only it satisfies the system

$$
\frac{\partial F}{\partial Z^{*}}=\frac{\partial F}{\partial Z^{\dagger}}=\frac{\partial F}{\partial \tilde{Z}}=0 .
$$

We recall Theorems 15.3 and 15.5 of [13], but see also [6]:

Theorem 2.9 Let $U \subseteq \mathbb{B} \mathbb{C}$ be a domain, and let $U_{\ell}, \ell=1,2$, be as in Remark 2.3. If $F_{1}$ and $F_{2}$ are complex holomorphic functions on $U_{1}$ and $U_{2}$, respectively, then the function:

$$
F(Z):=F\left(z+\mathbf{i}_{2} w\right)=\mathbf{e}_{1} F_{1}\left(z-\mathbf{i}_{1} w\right)+\mathbf{e}_{2} F_{2}\left(z+\mathbf{i}_{1} w\right),
$$

defined on $U$, is bicomplex holomorphic.

This description is in fact a characterization of bicomplex holomorphic functions.

Theorem 2.10 Let $U \subseteq \mathbb{B} \mathbb{C}$ be a domain and let $F$ be a bicomplex holomorphic function defined on $U$. Then there exist two complex holomorphic functions $F_{1}$ and $F_{2}$ defined on $U_{1}$ and $U_{2}$, as in Remark 2.3, such that

$$
F(Z)=F\left(z+\mathbf{i}_{2} w\right)=\mathbf{e}_{1} F_{1}\left(z-\mathbf{i}_{1} w\right)+\mathbf{e}_{2} F_{2}\left(z+\mathbf{i}_{1} w\right),
$$

for all $Z$ in $U$. 
In order to state the Cauchy's integral formula, we need some more notation: let $a=a_{1}+\mathbf{i}_{2} a_{2} \in \mathbb{B C}$ and let $r_{1}, r_{2}$ be positive numbers. We define the bi-disk $D\left(a, r_{1}, r_{2}\right)$ with center $a$ and radii $r_{1}, r_{2}$ as the set

$$
D\left(a, r_{1}, r_{2}\right)=\left\{z_{1}+\mathbf{i}_{2} z_{2} \in \mathbb{B} \mathbb{C}:\left|\left(z_{1}-\mathbf{i}_{1} z_{2}\right)-\left(a_{1}-\mathbf{i}_{1} a_{2}\right)\right|<r_{1},\left|\left(z_{1}+\mathbf{i}_{1} z_{2}\right)-\left(a_{1}+\mathbf{i}_{1} a_{2}\right)\right|<r_{2}\right\} .
$$

Let $C_{1}$ be the circle in $A_{1}$ with center at $a_{1}-\mathbf{i}_{1} a_{2}$ and radius $r_{1}$, and $C_{2}$ be the circle in $A_{2}$ with center at $a_{1}+\mathbf{i}_{1} a_{2}$ and radius $r_{2}$. Then $C=C_{1} \times C_{2}$ lies in the boundary of $D\left(a, r_{1}, r_{2}\right)$.

Theorem 2.11 (Cauchy's integral formula) Let $U \subset \mathbb{B C}$ be a domain and let $f: U \subset \mathbb{B C} \rightarrow \mathbb{B C}$ be a bicomplex holomorphic function. Assume that $a=a_{1}+\mathbf{i}_{2} a_{2} \in U$ and that the closure of the bi-disk $D\left(a, r_{1}, r_{2}\right)$, for suitable $r_{1}, r_{2}$, is contained in $U$. Let $C$ be as above and $w_{1}+\mathbf{i}_{2} w_{2} \in D\left(a, r_{1}, r_{2}\right)$ then

$$
f\left(w_{1}+\mathbf{i}_{2} w_{2}\right)=\frac{1}{2 \pi \mathbf{i}_{1}} \int_{C} \frac{f\left(z_{1}+\mathbf{i}_{2} z_{2}\right) d\left(z_{1}+\mathbf{i}_{2} z_{2}\right)}{\left(z_{1}+\mathbf{i}_{2} z_{2}\right)-\left(w_{1}+\mathbf{i}_{2} w_{2}\right)} .
$$

This theorem is in [13], Theorem 40.1 and its proof shows that the following, more general, result holds (compare also with Example 40.4 in [13]):

Theorem 2.12 Let $U \subset \mathbb{B} \mathbb{C}$ be a domain and let $f: U \subset \mathbb{B C} \rightarrow \mathbb{B C}$ be a bicomplex holomorphic function. Let $w_{1}+\mathbf{i}_{2} w_{2} \in U$ and let $\gamma_{1} \subset A_{1}$ and $\gamma_{2} \subset A_{2}$ be simple, positively oriented, closed curves which are union of continuously differentiable Jordan curves and such that $\gamma_{1}$ surrounds $w_{1}-\mathbf{i}_{1} w_{2}$ and $\gamma_{2}$ surrounds $w_{1}+\mathbf{i}_{1} w_{2}$. Assume that $\Gamma=\gamma_{1} \times \gamma_{2} \subset U$. Then:

$$
f\left(w_{1}+\mathbf{i}_{2} w_{2}\right)=\frac{1}{2 \pi \mathbf{i}_{1}} \int_{\Gamma} \frac{f\left(z_{1}+\mathbf{i}_{2} z_{2}\right) d\left(z_{1}+\mathbf{i}_{2} z_{2}\right)}{\left(z_{1}+\mathbf{i}_{2} z_{2}\right)-\left(w_{1}+\mathbf{i}_{2} w_{2}\right)} .
$$

Remark 2.13 The integral in (2.5) can be computed as follows:

$$
f\left(w_{1}+\mathbf{i}_{2} w_{2}\right)=\frac{1}{2 \pi \mathbf{i}_{1}} \int_{\gamma_{1}} \frac{f\left(z_{1}-\mathbf{i}_{1} z_{2}\right) d\left(z_{1}-\mathbf{i}_{1} z_{2}\right)}{\left(z_{1}-\mathbf{i}_{1} z_{2}\right)-\left(w_{1}-\mathbf{i}_{1} w_{2}\right)} \mathbf{e}_{1}+\frac{1}{2 \pi \mathbf{i}_{1}} \int_{\gamma_{2}} \frac{f\left(z_{1}+\mathbf{i}_{1} z_{2}\right) d\left(z_{1}+\mathbf{i}_{1} z_{2}\right)}{\left(z_{1}+\mathbf{i}_{1} z_{2}\right)-\left(w_{1}+\mathbf{i}_{1} w_{2}\right)} \mathbf{e}_{2} .
$$

\section{Bounded linear operators and the $\mathbb{B C}$-functional calculus}

In this paper, even when we do not specify it explicitly, we always limit ourselves to the case of bounded linear operators.

Let $V$ be a module over $\mathbb{B} \mathbb{C}$ and let $T: V \rightarrow V$ be a map such that

$$
\begin{aligned}
& T(v+u)=T(v)+T(u), \quad \forall v, u \in V, \\
& T(a v)=a T(v), \quad \forall v \in V, \forall a \in \mathbb{B} \mathbb{C} .
\end{aligned}
$$

Then we say that $T$ is a $\mathbb{B C}$-linear operator on $V$. The set $\operatorname{End}(V)$ of linear operators on $V$ forms a $\mathbb{B C}$-module by defining

$$
\begin{aligned}
& (T+S)(v)=T(v)+S(v), \quad T, S \in \operatorname{End}(V), \\
& (a T)(v)=a(T(v)), \quad T \in \operatorname{End}(V), \quad a \in \mathbb{B} \mathbb{C} .
\end{aligned}
$$

We can define the multiplication of operators and, in particular, the powers $T^{n}$. We set $T^{0}=I$ where $I$ denotes the identity operator.

Let us set $V_{1}:=\mathbf{e}_{1} V$ and $V_{2}:=\mathbf{e}_{2} V$. Any element $v \in V$ can be written as

$$
v=\left(\mathbf{e}_{1}+\mathbf{e}_{2}\right) v=v_{1}+v_{2}=\mathbf{e}_{1} v_{1}+\mathbf{e}_{2} v_{2},
$$

where $v_{\ell}=\mathbf{e}_{\ell} v$. It is immediate that $V=V_{1} \oplus V_{2}$ as $\mathbb{B C}$-module but also as $\mathbb{C}_{\mathbf{i}_{1}}$-linear space or $\mathbb{C}_{\mathbf{i}_{2}}$-linear space.

Definition 3.1 We can define the operators $T_{1}, T_{2}$ as (see [3])

$$
T_{\ell}(v):=\mathbf{e}_{\ell} T(v), \quad T_{\ell}: V \rightarrow V_{\ell}, \quad \ell=1,2 .
$$


In particular, we will use the two operators

$$
I_{\ell}(v):=\mathbf{e}_{\ell} I(v), \quad \ell=1,2 .
$$

Proposition 3.2 The following properties holds.

(1) The operators $T_{1}$ and $T_{2}$ are $\mathbb{B} \mathbb{C}$-linear.

(2) We have the decomposition

$$
T=\mathbf{e}_{1} T_{1}+\mathbf{e}_{2} T_{2} .
$$

(3) The action of $T$ on $V$ can be decomposed as follows

$$
T(v)=\mathbf{e}_{1} T_{1}\left(v_{1}\right)+\mathbf{e}_{2} T_{2}\left(v_{2}\right),
$$

where $v \in V, v=\mathbf{e}_{1} v_{1}+\mathbf{e}_{2} v_{2}$.

(4) The operator $T_{\ell}$ maps $V_{\ell}$ to itself, i.e.

$$
T_{\ell}: V_{\ell} \rightarrow V_{\ell}, \quad \ell=1,2 .
$$

In particular the operators $I_{\ell}: V_{\ell} \rightarrow V_{\ell}$ are the identities on $V_{\ell}, \ell=1,2$.

Pr o o f. Point (1) it is true since $T$ is $\mathbb{B C}$-linear. Point (2) follows from

$$
T=\mathbf{e}_{1} T+\mathbf{e}_{2} T=T_{1}+T_{2}=\mathbf{e}_{1} T_{1}+\mathbf{e}_{2} T_{2} .
$$

Point (3). The action of $T$ on $V$ can be decomposed using the actions of $T_{\ell}$ on $V_{\ell}, \ell=1,2$, their linearity and the fact that $\mathbf{e}_{1} \mathbf{e}_{2}=0$, as

$$
T(v)=\left(\mathbf{e}_{1} T_{1}+\mathbf{e}_{2} T_{2}\right)\left(\mathbf{e}_{1} v_{1}+\mathbf{e}_{2} v_{2}\right)=\mathbf{e}_{1} T_{1}\left(v_{1}\right)+\mathbf{e}_{2} T_{2}\left(v_{2}\right) .
$$

Point (4). The statement follows from

$$
T_{1}\left(\mathbf{e}_{1} v_{1}+\mathbf{e}_{2} v_{2}\right)=\mathbf{e}_{1} T_{1}\left(\mathbf{e}_{1} v_{1}+\mathbf{e}_{2} v_{2}\right)=\mathbf{e}_{1} T_{1}\left(v_{1}\right) .
$$

The case of $T_{2}$ is similar.

Definition 3.3 The decomposition (3.1) is called idempotent decomposition of the operator $T$.

Remark 3.4 If $T$ is an $m \times n$ matrix with bicomplex entries, then the idempotent decomposition can be further strengthened. In fact we have $T=\left[t_{i j}\right]$ with $t_{i j} \in \mathbb{B C}$ so $t_{i j}=\mathbf{e}_{1} t_{i j}^{1}+\mathbf{e}_{2} t_{i j}^{2}, t_{i j}^{\ell} \in A_{\ell}, \ell=1,2$. Thus we can write $T=\mathbf{e}_{1} \widetilde{T}_{1}+\mathbf{e}_{2} \widetilde{T}_{2}$ where $\widetilde{T}_{\ell}, \ell=1,2$, have complex entries. A similar statement is true, in particular, for the elements in $V$ when $V=\mathbb{B C}^{m \times 1}, V=\mathbb{B C}^{1 \times n}$ or when $V$ is finite dimensional (in this case it is sufficient to use the isomorphism $V \cong \mathbb{B C}^{m \times 1}$ where $m=\operatorname{dim} V$ ).

In the rest of the paper we assume that $V$ is a Banach $\mathbb{B C}$-module endowed with norm $\|\cdot\|$ and let $\mathcal{B}(V)$ be the $\mathbb{B C}$-module of the bounded operators acting on $V$. Here we consider $\|\cdot\|=\frac{1}{\sqrt{2}}\left(\|\cdot\|_{V_{1}}^{2}+\|\cdot\|_{V_{2}}^{2}\right)^{1 / 2}$ where $\|\cdot\|_{V_{\ell}}$ is the norm in $V_{\ell}$ considered as a $\mathbb{C}_{\mathbf{i}_{1}}$-linear space, $\ell=1,2$. The norm $\|\cdot\|$ is a real norm on the $\mathbb{B C}$-module $V$, seen as a real vector space, in the sense that for any $v \in V$ and $a \in \mathbb{B C}$ the inequality $\|a v\| \leq \sqrt{2}|a|\|v\|$ holds. As we shall see in the sequel, the fact that the norm is not multiplicative does not affect our results. For a complete discussion on the subtle issue of the norms in a $\mathbb{B C}$-module, we refer the read to [1], Section 4.1.

It is easy to verify that $\mathcal{B}(V)$ is a Banach $\mathbb{B C}$-module if we endow it with the norm

$$
\|T\|:=\sup _{v \in V, v \neq 0} \frac{\|T(v)\|}{\|v\|} .
$$

Definition 3.5 Let $T \in \mathcal{B}(V)$. We define the resolvent set $\rho(T)$ of $T$ as the subset of $\mathbb{B} \mathbb{C}$ for which the operator $(\lambda I-T)^{-1}$ exists as a linear bounded operator. The spectrum $\sigma(T)$ of $T$ is defined as the complement of $\rho(T)$, i.e.

$$
\sigma(T)=\{\lambda \in \mathbb{B C} \mid \lambda I-T \text { is not invertible in } \mathcal{B}(V)\} .
$$

The operator $R(\lambda, T)=(\lambda I-T)^{-1}$ is called the resolvent of $T$, for $\lambda \in \rho(T)$. 
In the case of bounded complex linear operators the spectrum is compact. Also for quaternionic, bounded linear operators (where the notion of spectrum has to be substituted by the notion of $S$-spectrum), we have that the $S$-spectrum is compact and non empty see [4]. In the bicomplex case this is not anymore true, as illustrated in the next example.

Example 3.6 Let us compute the spectrum of the identity matrix $I$ in $\mathbb{B} \mathbb{C}^{n \times n}$. This is the simplest case of matrix one may consider but it is useful to illustrate the situation in this setting. If $\lambda=\eta+\mathbf{i}_{2} \mu=\mathbf{e}_{1}(\eta-$ $\left.\mathbf{i}_{1} \mu\right)+\mathbf{e}_{2}\left(\eta+\mathbf{i}_{1} \mu\right)$, then $\lambda I-I=\mathbf{e}_{1}\left(\eta-1-\mathbf{i}_{1} \mu\right) I+\mathbf{e}_{2}\left(\eta-1+\mathbf{i}_{1} \mu\right) I$. The spectrum of $I$ coincides with the set of eigenvalues $\lambda$, so we need to look for the solutions of $(\lambda I-I) v=0, v=\mathbf{e}_{1} v_{1}+\mathbf{e}_{2} v_{2} \in \mathbb{B} \mathbb{C}^{n \times 1}$, which corresponds to the system

$$
\left(\eta-1-\mathbf{i}_{1} \mu\right) v_{1}=0, \quad\left(\eta-1+\mathbf{i}_{1} \mu\right) v_{2}=0, \quad v_{1}, v_{2} \in \mathbb{C}_{\mathbf{i}_{1}}^{n \times 1} .
$$

We easily obtain that the spectrum is the set

$$
\left\{\lambda \in \mathbb{B C} \mid \lambda=\mathbf{e}_{1} \cdot 1+\mathbf{e}_{2} \tilde{\mu}, \lambda=\tilde{\eta} \mathbf{e}_{1}+\mathbf{e}_{2} \cdot 1, \tilde{\eta}, \tilde{\mu} \in \mathbb{C}_{\mathbf{i}_{1}}\right\},
$$

where $\tilde{\mu}=1+2 \mathbf{i}_{1} \mu$ and $\tilde{\eta}=1-2 \mathbf{i}_{1} \mu$. The spectrum is not a compact set since it is unbounded.

In order to define a functional calculus we need a more refined description of the spectrum. We recall the following definition.

Definition 3.7 Let $T: V \rightarrow V$ be a $\mathbb{B C}$-linear operator. The spectrum $\sigma(T)$ decomposes into three disjoint sets:

(1) the point spectrum $\sigma_{p}(T)$ defined as

$$
\sigma_{p}(T)=\{\lambda \in \mathbb{B} \mathbb{C} \mid \operatorname{ker}(\lambda I-T) \neq\{0\}\} ;
$$

(2) the residual spectrum $\sigma_{r}(T)$ defined as

$$
\sigma_{r}(T)=\{\lambda \in \mathbb{B C} \mid \operatorname{ker}(\lambda I-T)=\{0\}, \overline{\operatorname{Ran}(\lambda I-T)} \neq V\} ;
$$

(3) the continuous spectrum $\sigma_{c}(T)$ defined as

$$
\sigma_{c}(T)=\left\{\lambda \in \mathbb{B C} \mid \operatorname{ker}(\lambda I-T)=\{0\}, \overline{\operatorname{Ran}(\lambda I-T)}=V,(\lambda I-T)^{-1} \notin \mathcal{B}(V)\right\} .
$$

We now describe more in detail the spectrum of a $\mathbb{B C}$-linear operator. To this end, we need the following result:

Proposition 3.8 Let $T: V \rightarrow V$ be a bicomplex linear operator. Then $T$ is bounded if and only if $T_{1}$ and $T_{2}$ are both bounded.

We omit the proof since the arguments are as in the proof of Theorem 3.1 in [3].

Lemma 3.9 Let $T: V \rightarrow V$ be a $\mathbb{B C}$-linear bounded operator and let $T=\mathbf{e}_{1} T_{1}+\mathbf{e}_{2} T_{2}$ be its idempotent decomposition. Then $T$ is invertible if and only if $T_{1}, T_{2}$ are invertible on $V_{1}, V_{2}$ respectively, and

$$
T^{-1}=\mathbf{e}_{1} T_{1}^{-1}+\mathbf{e}_{2} T_{2}^{-1} .
$$

Proof. Assume that $T$ is invertible, and denote by $S:=\mathbf{e}_{1} S_{1}+\mathbf{e}_{2} S_{2}$ the idempotent decomposition of its inverse. We have

$$
I=\mathbf{e}_{1} I_{1}+\mathbf{e}_{2} I_{2}=T\left(\mathbf{e}_{1} S_{1}+\mathbf{e}_{2} S_{2}\right)=\left(\mathbf{e}_{1} T_{1}+\mathbf{e}_{2} T_{2}\right)\left(\mathbf{e}_{1} S_{1}+\mathbf{e}_{2} S_{2}\right)=\mathbf{e}_{1} T_{1} S_{1}+\mathbf{e}_{2} T_{2} S_{2}
$$

from which we deduce $T_{\ell} S_{\ell}=I_{\ell}, \ell=1,2$, where $I_{\ell}$ is the identity operator acting on $V_{\ell}$ (note that the idempotent decomposition of an operator is obviously unique). Thus $T_{\ell}$ admits right inverse and so it is invertible on $V_{\ell}$ and $T_{\ell}^{-1}=S_{\ell}, \ell=1,2$. Conversely, if $T_{\ell}$ is invertible for $\ell=1,2$ we can consider the operator $\mathbf{e}_{1} T_{1}^{-1}+\mathbf{e}_{2} T_{2}^{-1}$. A simple computation shows that

$$
T\left(\mathbf{e}_{1} T_{1}^{-1}+\mathbf{e}_{2} T_{2}^{-1}\right)=\left(\mathbf{e}_{1} T_{1}+\mathbf{e}_{2} T_{2}\right)\left(\mathbf{e}_{1} T_{1}^{-1}+\mathbf{e}_{2} T_{2}^{-1}\right)=I
$$

and the statement follows. 
Theorem 3.10 Let $T: V \rightarrow V$ be a $\mathbb{B C}$-linear operator and let $T=\mathbf{e}_{1} T_{1}+\mathbf{e}_{2} T_{2}$ be its idempotent decomposition. Let $\sigma(T)$ be the spectrum of $T$ and let $\sigma\left(T_{\ell}\right)$ be the spectrum of $T_{\ell}: V_{\ell} \rightarrow V_{\ell}$ where $V_{\ell}=\mathbf{e}_{\ell} V, \ell=1,2$. Then, using the terminology in Definition 3.7, we have:

$$
\begin{aligned}
& \sigma_{p}(T)=\left(\mathbf{e}_{1} \sigma_{p}\left(T_{1}\right)+\mathbf{e}_{2} \mathbb{C}_{\mathbf{i}_{1}}\right) \cup\left(\mathbf{e}_{1} \mathbb{C}_{\mathbf{i}_{1}}+\mathbf{e}_{2} \sigma_{p}\left(T_{2}\right)\right), \\
& \sigma_{r}(T)=\left(\mathbf{e}_{1} \sigma_{r}\left(T_{1}\right)+\mathbf{e}_{2} \mathbb{C}_{\mathbf{i}_{1}}\right) \cup\left(\mathbf{e}_{1} \mathbb{C}_{\mathbf{i}_{1}}+\mathbf{e}_{2} \sigma_{r}\left(T_{2}\right)\right), \\
& \sigma_{c}(T)=\left(\mathbf{e}_{1} \sigma_{c}\left(T_{1}\right)+\mathbf{e}_{2} \mathbb{C}_{\mathbf{i}_{1}}\right) \cup\left(\mathbf{e}_{1} \mathbb{C}_{\mathbf{i}_{1}}+\mathbf{e}_{2} \sigma_{c}\left(T_{2}\right)\right),
\end{aligned}
$$

and, finally,

$$
\sigma(T)=\left(\mathbf{e}_{1} \sigma\left(T_{1}\right)+\mathbf{e}_{2} \mathbb{C}_{\mathbf{i}_{1}}\right) \cup\left(\mathbf{e}_{1} \mathbb{C}_{\mathbf{i}_{1}}+\mathbf{e}_{2} \sigma\left(T_{2}\right)\right) .
$$

Pro of. In the proof we consider the three subsets in which the spectrum decomposes and we divide the proof in steps.

Step 1. Let us consider the point spectrum. Let $\lambda \in \sigma_{p}(T)$ and let $\lambda=\lambda_{1} \mathbf{e}_{1}+\lambda_{2} \mathbf{e}_{2}, \lambda_{\ell} \in A_{\ell}, \ell=1$, 2. Then $\lambda I-T$ is not injective and so there exists a nonzero vector $v \in V$ such that $(\lambda I-T) v=0$. Using the idempotent decompositions obtain:

$$
\begin{aligned}
(\lambda I-T) v & =\left[\left(\mathbf{e}_{1} \lambda_{1}+\mathbf{e}_{2} \lambda_{2}\right)\left(\mathbf{e}_{1} I_{1}+\mathbf{e}_{2} I_{2}\right)-\left(\mathbf{e}_{1} T_{1}+\mathbf{e}_{2} T_{2}\right)\right]\left(\mathbf{e}_{1} v_{1}+\mathbf{e}_{2} v_{2}\right) \\
& =\left[\left(\mathbf{e}_{1} \lambda_{1} I_{1}+\mathbf{e}_{2} \lambda_{2} I_{2}\right)-\left(\mathbf{e}_{1} T_{1}+\mathbf{e}_{2} T_{2}\right)\right]\left(\mathbf{e}_{1} v_{1}+\mathbf{e}_{2} v_{2}\right) \\
& \left.=\left[\left(\mathbf{e}_{1} \lambda_{1} I_{1}-T_{1}\right) \mathbf{e}_{1}+\left(\mathbf{e}_{2} \lambda_{2} I_{2}-T_{2}\right) \mathbf{e}_{2}\right)\right]\left(\mathbf{e}_{1} v_{1}+\mathbf{e}_{2} v_{2}\right) \\
& =\left(\mathbf{e}_{1} \lambda_{1} I_{1}-T_{1}\right) \mathbf{e}_{1} v_{1}+\left(\mathbf{e}_{2} \lambda_{2} I_{2}-T_{2}\right) \mathbf{e}_{2} v_{2}=0
\end{aligned}
$$

which implies the two conditions

$$
\left(\mathbf{e}_{1} \lambda_{1} I_{1}-T_{1}\right) v_{1}=0, \quad\left(\mathbf{e}_{2} \lambda_{2} I_{2}-T_{2}\right) v_{2}=0 .
$$

Since $v \neq 0$ then at least one between $v_{1}$ and $v_{2}$ is not a zero vector. In the first case, we obtain that $\mathbf{e}_{1} \lambda_{1} \in \sigma\left(T_{1}\right)$ while $\lambda_{2}$ can be any number in $A_{2} \cong \mathbb{C}_{\mathbf{i}_{1}}$ since we can set $v_{2}=0$; in the second case, we obtain that $\mathbf{e}_{2} \lambda_{2} \in \sigma\left(T_{2}\right)$ while $\lambda_{1}$ can be any number in $A_{1} \cong \mathbb{C}_{\mathbf{i}_{1}}$. Note that an element in $\sigma\left(T_{\ell}\right)$ is of the form $\mathbf{e}_{\ell} \lambda_{\ell}$ and thus $\mathbf{e}_{\ell} \sigma\left(T_{\ell}\right)=$ $\sigma\left(T_{\ell}\right)$. So we have

$$
\sigma_{p}(T) \subseteq\left(\mathbf{e}_{1} \sigma_{p}\left(T_{1}\right)+\mathbf{e}_{2} \mathbb{C}_{\mathbf{i}_{1}}\right) \cup\left(\mathbf{e}_{1} \mathbb{C}_{\mathbf{i}_{1}}+\mathbf{e}_{2} \sigma_{p}\left(T_{2}\right)\right) .
$$

The opposite inclusion follows by an analogous reasoning, thus we conclude that (3.2) holds.

Step 2. Let us now consider the residual spectrum and let us assume that $\operatorname{ker}(\lambda I-T)=\{0\}$ but $\overline{\operatorname{Ran}(\lambda I-T)} \neq V$. Observe that for any operator $T=\mathbf{e}_{1} T_{1}+\mathbf{e}_{2} T_{2}$ it is $\operatorname{Ran}(T)=\operatorname{Ran}\left(\mathbf{e}_{1} T_{1}\right)+\operatorname{Ran}\left(\mathbf{e}_{2} T_{2}\right)$. So, the decompositions $T=\mathbf{e}_{1} T_{1}+\mathbf{e}_{2} T_{2}$ and $\lambda=\mathbf{e}_{1} \lambda_{1}+\mathbf{e}_{2} \lambda_{2}$, imply that the range of $\lambda I-T$ equals $\operatorname{Ran}\left(\mathbf{e}_{1} \lambda_{1} I_{1}-T_{1}\right)+\operatorname{Ran}\left(\mathbf{e}_{2} \lambda_{2} I_{2}-T_{2}\right)$ and

$$
\overline{\operatorname{Ran}(\lambda I-T)}=\overline{\operatorname{Ran}\left(\mathbf{e}_{1} \lambda_{1} I_{1}-T_{1}\right)}+\overline{\operatorname{Ran}\left(\mathbf{e}_{2} \lambda_{2} I_{2}-T_{2}\right)} .
$$

Thus $\overline{\operatorname{Ran}(\lambda I-T)} \neq V$ if and only if either

$$
\overline{\operatorname{Ran}\left(\mathbf{e}_{1} \lambda_{1} I_{1}-T_{1}\right)} \neq V_{1}, \quad \text { or } \quad \overline{\operatorname{Ran}\left(\mathbf{e}_{2} \lambda_{2} I_{2}-T_{2}\right)} \neq V_{2} .
$$

Assume that $\mathbf{e}_{1} \lambda_{1} \in \sigma_{r}\left(T_{1}\right)$; then $\overline{\operatorname{Ran}\left(\mathbf{e}_{1} \lambda_{1} I_{1}-T_{1}\right)} \neq V_{1}$ and thus $\overline{\operatorname{Ran}(\lambda I-T)} \neq V$. Similarly if we consider $\mathbf{e}_{2} \lambda_{2} \in \sigma_{r}\left(T_{2}\right)$. Conversely, if $\lambda \in \sigma_{r}(T)$ then either

$$
\overline{\operatorname{Ran}\left(\mathbf{e}_{1} \lambda_{1} I_{1}-T_{1}\right)} \neq V_{1} \quad \text { or } \quad \overline{\operatorname{Ran}\left(\mathbf{e}_{2} \lambda_{2} I_{2}-T_{2}\right)} \neq V_{2}
$$

and so the equality (3.3) holds. 
Step 3. Finally let us look at the continuous spectrum and let us assume that $\lambda \in \sigma_{c}(T)$, i.e. let $\lambda$ be such that $(\lambda I-T)^{-1} \notin \mathcal{B}(V)$. We have

$$
\lambda I-T=\mathbf{e}_{1}\left(\mathbf{e}_{1} \lambda_{1} I_{1}-T_{1}\right)+\mathbf{e}_{2}\left(\mathbf{e}_{2} \lambda_{2} I_{2}-T_{2}\right),
$$

where $\lambda=\mathbf{e}_{1} \lambda_{1}+\mathbf{e}_{2} \lambda_{2}$, and thus Lemma 3.9 imply that

$$
(\lambda I-T)^{-1}=\mathbf{e}_{1}\left(\mathbf{e}_{1} \lambda_{1} I_{1}-T_{1}\right)^{-1}+\mathbf{e}_{2}\left(\mathbf{e}_{2} \lambda_{2} I_{2}-T_{2}\right)^{-1} .
$$

From Proposition 3.8 we deduce that $(\lambda I-T)^{-1}$ is bounded if and only if $\left(\mathbf{e}_{1} \lambda_{1} I_{1}-T_{1}\right)^{-1}$, and $\left(\mathbf{e}_{2} \lambda_{2} I_{2}-T_{2}\right)^{-1}$ are both bounded. Thus $\lambda \in \sigma_{c}(T)$ if and only if either $\left(\mathbf{e}_{1} \lambda_{1} I_{1}-T_{1}\right)^{-1}$ or $\left(\mathbf{e}_{2} \lambda_{2} I_{2}-T_{2}\right)^{-1}$ are not bounded and this happens if and only if

$$
\lambda \in \mathbf{e}_{1} \sigma_{c}\left(T_{1}\right)+\mathbf{e}_{2} \mathbb{C}_{\mathbf{i}_{1}} \quad \text { or } \quad \lambda \in \mathbf{e}_{1} \mathbb{C}_{\mathbf{i}_{1}}+\mathbf{e}_{2} \sigma_{c}\left(T_{2}\right) .
$$

Thus we have proved (3.4).

Step 4. Since $\sigma(T)$ decomposes as $\sigma(T)=\sigma_{p}(T) \cup \sigma_{r}(T) \cup \sigma_{c}(T)$ and since (3.2), (3.3), (3.4) hold, then we have the statement.

The following is an immediate consequence of the previous theorem, and shows the general nature of Example 3.6:

Corollary 3.11 The spectrum $\sigma(T)$ of any operator $T \in \mathcal{B}(V)$ is unbounded.

Proof. It follows from the fact that $\sigma(T)=\left(\mathbf{e}_{1} \sigma\left(T_{1}\right)+\mathbf{e}_{2} \mathbb{C}_{\mathbf{i}_{1}}\right) \cup\left(\mathbf{e}_{1} \mathbb{C}_{\mathbf{i}_{1}}+\mathbf{e}_{2} \sigma\left(T_{2}\right)\right)$.

The proof of Theorem 3.10 also shows the following:

Corollary 3.12 Let $T \in \mathcal{B}(V)$, Then the spectrum of $T_{\ell}$ is contained in $\mathbf{e}_{\ell} \mathbb{C}_{\mathbf{i}_{1}}, \ell=1,2$.

It is now clear that the notion of spectrum is not useful to define a functional calculus, and we therefore introduce another notion of spectrum, which is better suited to our purposes:

Definition 3.13 We call reduced spectrum the set $\sigma_{\text {red }}(T)$ defined as:

$$
\sigma_{\text {red }}(T):=\left(\mathbf{e}_{1} \sigma\left(T_{1}\right)+\mathbf{e}_{2} \mathbb{C}_{\mathbf{i}_{1}}\right) \cap\left(\mathbf{e}_{1} \mathbb{C}_{\mathbf{i}_{1}}+\mathbf{e}_{2} \sigma\left(T_{2}\right)\right)=\mathbf{e}_{1} \sigma\left(T_{1}\right)+\mathbf{e}_{2} \sigma\left(T_{2}\right) .
$$

Example 3.14 In the case of the identity matrix, the reduced spectrum consists of the point $\mathbf{e}_{1}+\mathbf{e}_{2}$, i.e. the real number 1 .

Theorem 3.15 Let $T \in \mathcal{B}(V)$ and let $U \subset \mathbb{B} \mathbb{C}$ be a domain containing the reduced spectrum of $T$. Let $w_{1}+\mathbf{i}_{2} w_{2} \in U$ and let $\gamma_{1} \subset A_{1}$ and $\gamma_{2} \subset A_{2}$ be simple, positively oriented, closed curves which are unions of continuously differentiable Jordan curves and such that $\gamma_{1}$ surrounds $w_{1}-\mathbf{i}_{1} w_{2}, \gamma_{2}$ surrounds $w_{1}+\mathbf{i}_{1} w_{2}$, and $\Gamma=\gamma_{1} \times \gamma_{2} \subset U$. Then:

(1) Let $T=\mathbf{e}_{1} T_{1}+\mathbf{e}_{2} T_{2}$. Then the resolvent operator series $\sum_{n \geq 0} \lambda^{-n-1} T^{n}$ converges in the bi-disk $D\left(0, r_{1}, r_{2}\right)$ centered in the origin for $r_{1}>\left\|T_{1}\right\|$ and $r_{2}>\left\|T_{2}\right\|$.

(2) For every $m \in \mathbb{N} \cup\{0\}$ we have

$$
T^{m}=\frac{1}{2 \pi \mathbf{i}_{1}} \int_{\Gamma}(\lambda I-T)^{-1} \lambda^{m} d \lambda .
$$

(3) The reduced spectrum $\sigma_{\text {red }}(T)$ of $T$ is compact and nonempty.

Pr o of. Point (1). Using the idempotent decomposition of the operator $T$ and of $\lambda \in \mathbb{B C}$ we have

$$
\begin{aligned}
\sum_{n \geq 0} \lambda^{-n-1} T^{n} & =\sum_{n \geq 0}\left(\mathbf{e}_{1} \lambda_{1}+\mathbf{e}_{2} \lambda_{2}\right)^{-n-1}\left(\mathbf{e}_{1} T_{1}+\mathbf{e}_{2} T_{2}\right)^{n} \\
& =\mathbf{e}_{1} \sum_{n \geq 0} \mathbf{e}_{1} \lambda_{1}^{-n-1} T_{1}^{n}+\mathbf{e}_{2} \sum_{n \geq 0} \mathbf{e}_{2} \lambda_{2}^{-n-1} T_{2}^{n} .
\end{aligned}
$$


We have that $\sum_{n \geq 0} \mathbf{e}_{\ell} \lambda_{\ell}^{-n-1} T_{\ell}^{n}$ converges to $\left(\mathbf{e}_{\ell} \lambda_{\ell} I_{\ell}-T_{\ell}\right)^{-1}, \ell=1,2$, if and only if $r_{1}>\left\|T_{1}\right\|$ and $r_{2}>\left\|T_{2}\right\|$. Thus in $D\left(0, r_{1}, r_{2}\right)$ we have

$$
\sum_{n \geq 0} \lambda^{-n-1} T^{n}=\mathbf{e}_{1}\left(\mathbf{e}_{1} \lambda_{1} I_{1}-T_{1}\right)^{-1}+\mathbf{e}_{2}\left(\mathbf{e}_{2} \lambda_{2} I_{2}-T_{2}\right)^{-1}=(\lambda I-T)^{-1} .
$$

Point (2). We observe that, using point (1), the idempotent decomposition of the resolvent operator and of $\lambda=\mathbf{e}_{1} \lambda_{1}+\mathbf{e}_{2} \lambda_{2}$, we get

$$
\begin{aligned}
& \frac{1}{2 \pi \mathbf{i}_{1}} \int_{\Gamma}(\lambda I-T)^{-1} \lambda^{m} d \lambda \\
& \quad=\frac{1}{2 \pi \mathbf{i}_{1}} \mathbf{e}_{1} \sum_{n \geq 0} \int_{D_{1}(0)} \lambda_{1}^{-n-1} T_{1}^{n} \lambda_{1}^{m} d \lambda_{1}+\frac{1}{2 \pi \mathbf{i}_{1}} \mathbf{e}_{2} \sum_{n \geq 0} \int_{D_{2}(0)} \lambda_{2}^{-n-1} T_{2}^{n} \lambda_{2}^{m} d \lambda_{2} \\
& \quad=\frac{1}{2 \pi \mathbf{i}_{1}} \mathbf{e}_{1} T_{1}^{n} \sum_{n \geq 0} \int_{D_{1}(0)} \lambda_{1}^{-n-1} \lambda_{1}^{m} d \lambda_{1}+\frac{1}{2 \pi \mathbf{i}_{1}} \mathbf{e}_{2} T_{2}^{n} \sum_{n \geq 0} \int_{D_{2}(0)} \lambda_{2}^{-n-1} \lambda_{2}^{m} d \lambda_{2} \\
& \quad=\mathbf{e}_{1} T_{1}^{m}+\mathbf{e}_{2} T_{2}^{m} .
\end{aligned}
$$

Since $\mathbf{e}_{1} T_{1}^{m}+\mathbf{e}_{2} T_{2}^{m}=T^{m}$ we get the statement.

Point (3). For $m=0$ in Point (2) we have

$$
\frac{1}{2 \pi \mathbf{i}_{1}} \int_{\Gamma}(\lambda I-T)^{-1} d \lambda=I .
$$

which shows that $\sigma_{\text {red }}(T)$ is a nonempty set, otherwise the integral would be zero by the Cauchy's theorem. The reduced spectrum is closed because the complement of $\sigma_{\text {red }}(T)$ is open. Indeed, since

$$
\lambda I-T=\mathbf{e}_{1}\left(\mathbf{e}_{1} \lambda_{1} I_{1}-T_{1}\right)+\mathbf{e}_{2}\left(\mathbf{e}_{2} \lambda_{2} I_{2}-T_{2}\right),
$$

the function

$$
G: \mathbf{e}_{1} \lambda_{1}+\mathbf{e}_{2} \lambda_{2} \longmapsto \mathbf{e}_{1}\left(\mathbf{e}_{1} \lambda_{1} I_{1}-T_{1}\right)+\mathbf{e}_{2}\left(\mathbf{e}_{2} \lambda_{2} I_{2}-T_{2}\right)
$$

is trivially continuous and, by Theorem 10.12 in [17], the set $\mathcal{U}(V)$ of all invertible elements of $\mathcal{B}(V)=$ $\mathbf{e}_{1} \mathcal{B}(V)+\mathbf{e}_{2} \mathcal{B}\left(V_{2}\right)$ is an open set in $\mathcal{B}(V)$. Therefore $g^{-1}(\mathcal{U}(V))$ is an open set in $\mathbb{B C}$. The reduced spectrum is a bounded set because the series $\sum_{n \geq 0} T_{j}^{n} \lambda_{j}^{-1-n}, j=1,2$, converges for $\left\|T_{j}\right\|<\left|\lambda_{j}\right|, j=1,2$. So we conclude that it is compact.

In our next result we obtain an estimate on the spectral radius. First of all, we note that in order to define the functional calculus we used the reduced spectrum, thus it makes sense to define the (reduced) spectral radius as follows:

$$
r(T):=\sup \left\{|\lambda| \mid \lambda \in \sigma_{\text {red }}(T)\right\} .
$$

We have the following result:

Proposition 3.16 (Spectral radius) Let $V$ be a Banach module over $\mathbb{B C}$ and let $T: V \rightarrow V$ be a $\mathbb{B} \mathbb{C}$-linear bounded operator. The spectral radius $r(T)$ satisfies

$$
r(T) \leq \frac{\sqrt{2}}{2}\left(r\left(T_{1}\right)+r\left(T_{2}\right)\right)
$$

Proof. Since $\lambda=\mathbf{e}_{1} \lambda_{1}+\mathbf{e}_{2} \lambda_{2}$ we obviously have that $|\lambda| \leq \frac{\sqrt{2}}{2}\left(\left|\lambda_{1}\right|+\left|\lambda_{2}\right|\right)$ and so $r(T) \leq \frac{\sqrt{2}}{2}\left(r\left(T_{1}\right)+\right.$ $\left.r\left(T_{2}\right)\right)$.

Theorem 3.17 Let $\lambda \in \rho(T)$ and consider the idempotent decomposition of the resolvent operator

$$
(\lambda I-T)^{-1}=\mathbf{e}_{1}\left(\mathbf{e}_{1} \lambda_{1} I_{1}-T_{1}\right)^{-1}+\mathbf{e}_{2}\left(\mathbf{e}_{2} \lambda_{2} I_{2}-T_{2}\right)^{-1} .
$$

Then we have 
(1) For $\lambda, \mu \in \rho(T)$

$$
(\lambda I-T)^{-1}-(\mu I-T)^{-1}=-(\lambda-\mu)(\lambda I-T)^{-1}(\mu I-T)^{-1} .
$$

(2) For $\lambda, \mu \in \rho(T)$

$$
(\lambda I-T)^{-1}(\mu I-T)^{-1}=(\mu I-T)^{-1}(\lambda I-T)^{-1} .
$$

(3) For $\lambda, \mu \in \rho(T)$

$$
\begin{aligned}
& \left(\mathbf{e}_{\ell} \lambda_{\ell} I_{\ell}-T_{\ell}\right)^{-1}-\left(\mathbf{e}_{\ell} \mu_{\ell} I_{\ell}-T_{\ell}\right)^{-1} \\
& \quad=-\left(\lambda_{\ell}-\mu_{\ell}\right)\left(\mathbf{e}_{\ell} \lambda_{\ell} I_{\ell}-T_{\ell}\right)^{-1}\left(\mathbf{e}_{\ell} \mu_{\ell} I_{\ell}-T_{\ell}\right)^{-1}, \quad \ell=1,2 .
\end{aligned}
$$

(4) For $\lambda, \mu \in \rho(T)$

$$
\left(\mathbf{e}_{\ell} \lambda_{\ell} I_{\ell}-T_{\ell}\right)^{-1}\left(\mathbf{e}_{\ell} \mu_{\ell} I_{\ell}-T_{\ell}\right)^{-1}=\left(\mathbf{e}_{\ell} \mu_{\ell} I_{\ell}-T_{\ell}\right)^{-1}\left(\mathbf{e}_{\ell} \lambda_{\ell} I_{\ell}-T_{\ell}\right)^{-1}, \quad \ell=1,2 .
$$

Pro of. Points (1) and (2) can be deduced by standard computations as in the complex case. Points (3) and (4) follow from (1) and (2) and from the idempotent decomposition since

$$
\begin{aligned}
& (\lambda I-T)^{-1}(\mu I-T)^{-1} \\
& \quad=\mathbf{e}_{1}\left(\mathbf{e}_{1} \lambda_{1} I_{1}-T_{1}\right)^{-1}\left(\mathbf{e}_{1} \mu_{1} I_{1}-T_{1}\right)^{-1}+\mathbf{e}_{2}\left(\mathbf{e}_{2} \lambda_{2} I_{2}-T_{1}\right)^{-1}\left(\mathbf{e}_{2} \mu_{2} I_{2}-T_{1}\right)^{-1} .
\end{aligned}
$$

Definition 3.18 We will denote by $\mathcal{H}(T)$ the set of functions which are bicomplex holomorphic in a neighborhood of $\sigma_{\text {red }}(T)$.

We now proof our main results

Theorem 3.19 Let $V$ be a Banach module over $\mathbb{B C}$ and let $T \in \mathcal{B}(V)$. Let $f \in \mathcal{H}(T)$, and let $D\left(a_{1}+\mathbf{i}_{2} a_{2} ; r_{1}, r_{2}\right)$ be a bi-disk whose closure is contained in an open set $U \supset \sigma_{\text {red }}(T)$ on which $f$ is bicomplex holomorphic. Let $\gamma_{\ell}$, be union of continuously differentiable Jordan curves for $\ell=1,2$ and such that $\Gamma=\gamma_{1} \times \gamma_{2}$ is contained in $D\left(a, r_{1}, r_{2}\right)$. Then the operator

$$
\frac{1}{2 \pi \mathbf{i}_{1}} \int_{\Gamma} f(\lambda)(\lambda I-T)^{-1} d \lambda
$$

does not depend on the choice of $\Gamma$.

Proof. By Theorem 2.10, there exist $f_{1}, f_{2}$ complex holomorphic such that

$$
f(\lambda)=\mathbf{e}_{1} f_{1}\left(\lambda_{1}\right)+\mathbf{e}_{2} f_{2}\left(\lambda_{2}\right)
$$

where

$$
\lambda=\mathbf{e}_{1} \lambda_{1}+\mathbf{e}_{2} \lambda_{2},
$$

and so

$$
d \lambda=\mathbf{e}_{1} d \lambda_{1}+\mathbf{e}_{2} d \lambda_{2} .
$$

Moreover,

$$
(\lambda I-T)^{-1}=\mathbf{e}_{1}\left(\lambda_{1} \mathbf{e}_{1} I_{1}-T_{1}\right)^{-1}+\mathbf{e}_{2}\left(\lambda_{2} \mathbf{e}_{2} I_{2}-T_{2}\right)^{-1}, \quad \lambda \in \rho(T),
$$

thus

$$
f(\lambda)(\lambda I-T)^{-1} d \lambda=\mathbf{e}_{1} f_{1}\left(\lambda_{1}\right)\left(\lambda_{1} \mathbf{e}_{1} I_{1}-T_{1}\right)^{-1} d \lambda_{1}+\mathbf{e}_{2} f_{2}\left(\lambda_{2}\right)\left(\lambda_{2} \mathbf{e}_{1} I_{2}-T_{2}\right)^{-1} d \lambda_{2},
$$

and

$$
\begin{aligned}
& \frac{1}{2 \pi \mathbf{i}_{1}} \int_{\Gamma} f(\lambda)(\lambda I-T)^{-1} d \lambda \\
& \quad=\frac{1}{2 \pi \mathbf{i}_{1}} \mathbf{e}_{1} \int_{\gamma_{1}} f_{1}\left(\lambda_{1}\right)\left(\mathbf{e}_{1} \lambda_{1} I_{1}-T_{1}\right)^{-1} d \lambda_{1}+\frac{1}{2 \pi \mathbf{i}_{1}} \mathbf{e}_{2} \int_{\gamma_{2}} f_{2}\left(\lambda_{2}\right)\left(\mathbf{e}_{2} \lambda_{2} I_{2}-T_{2}\right)^{-1} d \lambda_{2}
\end{aligned}
$$


The two integrands are singular at the points belonging to $\sigma\left(T_{1}\right)$ and $\sigma\left(T_{2}\right)$, respectively. Since $\Gamma$ properly contains the reduced spectrum, the integrand is continuous and thus the integral is well defined and defines a $\mathbb{B C}$-linear bounded operator. Let us consider a linear functional $\phi \in V^{\prime}$ where $V^{\prime}$ denotes the dual of $V$ and, for $v \in V$, consider the function

$$
g(\lambda):=\left\langle\phi,(\lambda I-T)^{-1} v\right\rangle .
$$

It is immediate to verify that the function $g(\lambda)$ is bicomplex holomorphic in $\lambda$, for $\lambda \in \rho(T)$. Note that $g(\lambda) \rightarrow 0$ for $\lambda \rightarrow \infty$ so $g$ is bicomplex holomorphic also at infinity. The fact that the integral

$$
\frac{1}{2 \pi \mathbf{i}_{1}} \int_{\Gamma} f(\lambda) g(\lambda) d \lambda
$$

does not depend on $\Gamma$ follows by the Cauchy theorem, see [13]. Thanks to the Hahn-Banach theorem (see Theorem $4.8,[9])$ we conclude the proof.

We can now give the following:

Definition 3.20 Let $V$ be a Banach module over $\mathbb{B C}, T \in \mathcal{B}(V)$ and $\Gamma$ as in Theorem 3.19. For any function $f$, bicomplex holomorphic in a neighborhood of $\sigma_{\text {red }}(T)$, we define

$$
f(T)=\frac{1}{2 \pi \mathbf{i}_{1}} \int_{\Gamma} f(\lambda)(\lambda I-T)^{-1} d \lambda .
$$

We have the following algebraic properties:

Proposition 3.21 Let $V$ be a $\mathbb{B} \mathbb{C}$-module and let $T \in \mathcal{B}(V)$. Let $f, g$ be two functions locally bicomplex holomorphic on the reduced spectrum of $T$. Then:

(1) $(f+g)(T)=f(T)+g(T)$;

(2) $($ af $)(T)=a f(T)$, for all $a \in \mathbb{B} \mathbb{C}$,

(3) $(f g)(T)=f(T) g(T)$,

(4) If $f(Z)=\sum_{j=0}^{\infty} a_{j} Z^{j}$ on a suitable bi-disk, then $f(T)=\sum_{j=0}^{\infty} a_{j} T^{j}$.

Pr o o f. Points (1) and (2) are immediate. Point (3) follows from the classical case of holomorphic functional calculus and from Theorem 3.17. Point (4) follows from the definition.

\section{References}

[1] D. Alpay, M. E. Luna-Elizarraras, M. Shapiro, and D. C. Struppa, Basics of functional analysis with bicomplex scalars, and bicomplex Schur analysis (Springer, to appear 2014), arXiv:1304.0781.

[2] H. M. Campos and V. V. Kravchenko, Fundamentals of bicomplex pseudoanalytic function theory: Cauchy integral formulas, negative formal powers and Schrödinger equations with complex coefficients, Complex Anal. Oper. Theory 7, 485-518 (2013).

[3] K. S. Charak, R. Kumar, and D. Rochon, Infinite dimensional bicomplex spectral decomposition theorem, Adv. Appl. Clifford Algebr. 23, 593-605 (2013).

[4] F. Colombo, I. Sabadini, and D. C. Struppa, Noncommutative Functional Calculus, Theory and Applications of Slice Hyperholomorphic Functions, Progress in Mathematics Vol. 289 (Birkhäuser Springer Basel AG, Basel, 2011).

[5] F. Colombo, I. Sabadini, D. C. Struppa, A. Vajiac, and M. Vajiac, Singularities of functions of one and several bicomplex variables, Ark. Mat. 49, 277-294 (2011).

[6] F. Colombo, I. Sabadini, D. C. Struppa, A. Vajiac, and M. Vajiac, Bicomplex hyperfunctions, Ann. Mat. Pura Appl. 190, 247-261 (2011).

[7] H. De Bie, D. C. Struppa, A. Vajiac, and M. B. Vajiac, The Cauchy-Kowalewski product for bicomplex holomorphic functions, Math. Nachr. 285, 1230-1242 (2012).

[8] B. Jefferies, Spectral Properties of Noncommuting Operators, Lecture Notes in Mathematics Vol. 1843 (Springer-Verlag, Berlin, 2004).

[9] R. Kumar, R. Kumar, and D. Rochon, The fundamental theorems in the framework of bicomplex topological modules, arxiv: $1109.3424 \mathrm{v} 1$. 
[10] M. E. Luna-Elizarraras, M. Shapiro, and D. C. Struppa, On Clifford analysis for holomorphic mappings, Adv. Geom. (2013), to appear.

[11] M. E. Luna-Elizarraras, M. Shapiro, D. C. Struppa, and A. Vajiac, Bicomplex numbers and their elementary functions, Cubo 14, 61-80 (2012).

[12] M. E. Luna-Elizarraras, M. Shapiro, D. C. Struppa, and A. Vajiac, Complex Laplacian and derivatives of bicomplex functions, Complex Anal. Oper. Theory 7, 1675-1711 (2013).

[13] G. B. Price, An Introduction to Multicomplex Spaces and Functions (Marcel Dekker, New York, 1991).

[14] D. Rochon and M. Shapiro, On algebraic properties of bicomplex and hyperbolic numbers, Anal. Univ. Oradea Fasc. Math. 11, 71-110 (2004).

[15] J. Ryan, Complexified Clifford analysis, Complex Var. Elliptic Eq. 1, 119-149 (1982).

[16] J. Ryan, $C^{2}$ extensions of analytic functions in the complex plane, Adv. Appl. Clifford Algebr. 11, 137-145 (2001).

[17] W. Rudin, Functional Analysis, McGraw-Hill Series in Higher Mathematics (McGraw-Hill Book Co., New YorkDüsseldorf-Johannesburg, 1973).

[18] G. Scorza Dragoni, Sulle funzioni olomorfe di una variabile bicomplessa, Reale Accad. dItalia, Mem. Classe Sci. Nat. Fis. Mat. 5, 597-665 (1934).

[19] N. Spampinato, Sulla rappresentazione di funzioni di variabile bicomplessa totalmente derivabili, Ann. Mat. Pura Appl. 14, 305-325 (1936). 